recognized weakness. This led to the establishment in 1969 of the Joint Organization for Solar Observations (JOSO), which has considerably increased the cooperation between the solar physicists. JOSO has tested some forty sites in southern Europe and has reached agreement on the superiority of the Canary Islands sites on La Palma and Tenerife. Phase A of the feasibility study for a large European solar telescope has recently been completed.

Solar radio astronomers have joined forces in the Committee for European Solar Radio Astronomy (CESRA) and are using to good effect the radio astronomy observing facilities within Europe. There is, however, no European equivalent of the Very Large Array in the USA.
The early rocket programmes led to a great interest in extreme ultra-violet and $\mathrm{X}$-ray spectroscopy in several countries, and it was possible to be involved in space projects even when the hardware was not developed in Europe. In most other disciplines of solar physics, European groups continue to maintain a high scientific standard.

\title{
Diagnostics of the Solar Interior
}

\author{
Douglas Gough, Cambridge \\ (Institute of Astronomy and Department of Applied Mathematics and Theoretical Physics)
}

Our basic ideas about the structure of the solar interior come mainly from theory, in which there are many simplifying approximations and assumptions. Some of these are well established, but others are quite uncertain. It is a task of the solar physicist to evaluate the implications of the uncertain assumptions and to attempt to test them by observation.

The most fundamental approximation is that the Sun is spherically symmetrical and in hydrostatic support. This is well borne out by observation, and is an extremely accurate first approximation for determining the vertical balance of forces. Any deviation from hydrostatic support would lead to a dynamical relaxation on a timescale of an hour or less, and the Sun is observed not to change its shape or size on that timescale to about one part in $10^{5}$. Low amplitude oscillations are observed, however; their most immediate importance is their diagnostic power, which is discussed below.

The horizontal balance of forces is quite a different matter. It was shown early in this century by Eddington and von Zeipel that unless a force or acceleration that breaks spherical symmetry has a very special form, it cannot find a reaction to balance it. Therefore it would induce material motion. This is true of rotation, for example, which induces large-scale circulation. The consequences of that circulation are difficult to ascertain, but there is bound to be some redistribution of the chemical elements and angular momentum.

In the theory of the evolution of solartype stars, material mixing by circulation currents is normally ignored. Thus, for example, unless there is thermal convection, the products of nuclear reactions are assumed to remain in situ. This is crucial to evaluating the subsequent evolution of the star, in particular, the rate at which a star evolves from the main sequence once hydrogen is exhausted from its centre. It is evident that if the hydrogen fuel were replenished from the cooler envelope by mixing, the main-sequence lifetime of that star would be prolonged. The ages of globular clusters, which are used in estimating the ages of galaxies, are measured by comparing with theory the observed turnoff from the main sequence. Thus our astronomical timescale is intimately linked with the assumptions upon which the theory of stellar evolution depends. It is important, therefore, that the predictions of that theory be tested as carefully as possible, and at present our best opportunity for really delicate testing is comparison with the Sun.

The structure of a star is not determined solely by the dynamical forces. It depends also on the thermal balance. Thus it is necessary to know how thermal energy is created and transported. This, in turn, requires knowledge of the nuclear reaction rates and opacities, and a theory of convection. It is also necessary to know the equation of state. Clearly, any model of the Sun must produce the observed photon luminosity and provide a flux of neutrinos that does not exceed the upper bound, set by observation.

\section{Calibration of Standard Solar Models}

It is usual to consider as model, a theoretical stellar body of the same mass as the Sun that, from an intitially homogeneous chemical state, evolves with time owing to the nuclear transmutations in the core. The initial chemical abundances $X, Y$ and $Z$ of hydrogen, helium and heavy elements are in some sense undetermined parameters, though one does have some idea of their values. The relative abundances of the heavy elements are usually taken from spectroscopic observations of the solar photosphere and measurements of meteoritic abundances. As $Y+Y+Z$ $=1$, only two of these parameters are independent. There is also at least one, and usually only one, explicitly arbitrary parameter in the formula for the convective heat flux $(\alpha)$. Reynolds stresses are usually ignored.

The parameters $Z$ and $Y$ (say) and $\alpha$ are adjusted so as to give the correct luminosity $L$ and radius $R$ of the Sun now. As there are three parameters to adjust for only two observations, a one-parameter sequence of models results.

Models of the present Sun have a radiative interior and a convective envelope. In practice, as the mixing-length parameter $\alpha$ affects directly only the outer convective envelope, which contains very little mass, conditions in the interior are hardly influenced by it. Thus, roughly, $\alpha$ determines $R$, and $L$ is determined by the chemical composition. It is best, therefore, to label the sequence of models by a chemical abundance, and here I choose $Z$. It is thought that the best value of $Z$ is about 0.018 , which yields $Y \cong 0.25$.

The time taken for the Sun to approach thermal balance and the time for the principal energy generating nuclear reactions to reach local equilibrium are short compared with the Sun's age. Moreover, the model of the present Sun is quite insensitive to the thermal structure adopted as initial conditions. Consequently it does not matter if the initial temperature distribution does not faithfully represent our ideas of what conditions were actually like when the Sun arrived at the main sequence.

Aside from a boundary layer only $10^{3} \mathrm{~km}$ thick, the convective envelope is very close to being adiabatically stratified. Thus the purpose of the calibration of $\alpha$ is simply to attach the photosphere, through that boundary layer, to that adiabat which matches smoothly onto the radiative interior.

The evolution of the models is such as to lead to a gradual rise in luminosity with time from an initial value of about 70 per cent of the present value. Radius changes are smaller, and depend on the convection theory adopted: an increase of about $15 \%$ is not atypical. The models appear to be stable to thermal instabilities, and as the thermal relaxation time is much less than the nuclear evolution time, a thermal balance between the energy generation rate in the core and the luminosity at the photosphere is accurately maintained.

\section{Solar Neutrino Problem}

The description above summarizes briefly the evolution of what is called the standard solar model. Most stellar physicists probably believe that it is quite an accurate first-order representation of the actual Sun. Nevertheless, as is now widely known, when a plausible value of $Z$ is 
adopted, the model fails to predict a neutrino flux compatible with observation. The theoretical value exceeds the measurements by a factor of about three. This is an issue of great importance, for it is telling us either that something is seriously wrong with the astrophysical assumptions upon which the standard theory depends, or that the atomic or nuclear physics used in computing opacities or nuclear reaction rates is substantially in error. It is perhaps unfortunate that the main contribution to the predicted neutrino flux, as measured currently, comes from a rare temperaturesensitive side reaction to the proton-proton chain that produces ${ }^{8} \mathrm{~B}$, which hardly contributes to the thermal energy: the present neutrino data do not provide a direct measurement of the reaction rates in the energetically significant parts of the chain.

One way of testing the theory is to measure neutrinos with detectors that are sensitive to the low-energy neutrinos produced in the main chains. Such experiments are planned. In particular one might hope to find out whether the model and the nuclear physics upon which it is based are at least approximately correct, and whether the thermonuclear energy generation rate $L_{n}$ is at least roughly in balance with the luminosity $L$. It is unlikely that the measurements will be sensitive enough, at least at first, to detect differences between $L$ and $L_{n}$ as low as $5 \%$, such as have been suggested to have arisen as a result of intermittent instabilities in the Sun's core. Differences of this order could be associated with a reduction in the ${ }^{8} \mathrm{~B}$ neutrino flux by a factor three, and so account for a substantial fraction of the current discrepancy between theory and observation.

A potentially more powerful way of testing the theory is to analyse the frequencies of low-amplitude oscillation that the Sun is observed to undergo. In principle, these could give us valuable information about the stratification of the Sun.

\section{Five-minute Oscillations of High De- gree}

Oscillation eigenmodes are classified by their order and degree, which are analogous to the radial and angular-momentum quantum numbers in the theory of the hydrogen atom. These parameters can be considered as measures of the vertical and horizontal components of the wave number vector.

The most important oscillations of high degree are standing acoustic waves trapped in a waveguide beneath the photosphere. Provided the circular frequency $v$ does not exceed about $5 \mathrm{mHz}$, the solar atmosphere acts as a reflecting boundary. When $v \geq 5 \mathrm{mHz}$ the vertical wavelength of a sound wave is less than the scale height of the atmosphere, and the wave can propagate into the chromosphere and corona. Otherwise the atmosphere is simp-

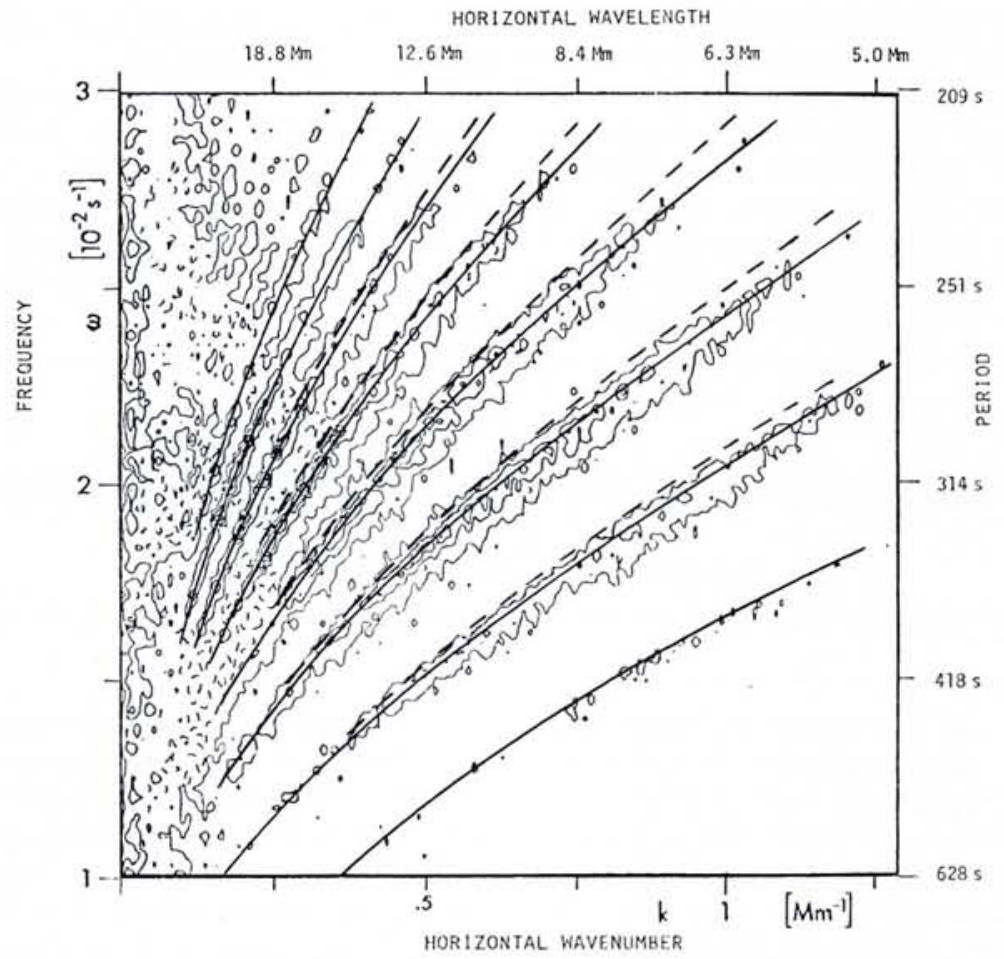

Fig. 1 - Two-dimensional power spectrum of the high-degree five-minute oscillations (from Deubner F.-L., Ulrich R.K. and Rhodes Jr. E.J., 1979, Astron. Astrophys., 72 (1979) 177). The continuous line represents the theoretical dispersion relation for a convection zone $2.2 \times 10^{5} \mathrm{~km}$ deep with $Z=0.02$ and $Y=0.25$; the dashed line is for a convection zone $1.5 \times 10^{5} \mathrm{~km}$ deep, with $Z=0.004$ and $Y=0.19$.

Iy lifted up and down, and does not permit the wave to propagate. The wave is refracted at depth, due to the rise in the sound speed. Thus an acoustical cavity is formed, whose dimensions depend on the frequency and horizontal wavenumber of the wave.

It has been possible to measure the time dependence and spatial dependence (in one dimension) of the oscillations, and thus to compute a two-dimensional power spectrum which measures the dispersion relation of the trapped waves. This was first achieved by F.-L. Deubner, who is now at the University of Würzburg. The measurements have been compared with theory, notably by G. Berthomieu and her colleagues at the Nice Observatory and by S. Lubow, E.J. Rhodes Jr. and R.K. Ulrich at the University of California, Los Angeles. They found that the most important quantity on which the eigenfrequencies depend is the pressure-density relation in the adiabatically stratified part of the convection zone, where most of the energy in the modes resides.

The result of the comparison with observation is a determination of the convective adiabat which, if extrapolated to the point of convective stability, yields a convection zone about $2 \times 10^{5} \mathrm{~km}$ deep. In recent years it had been thought that the convection zone is somewhat shallower than that, perhaps because models with deep convection zones predict high neutrino fluxes.

Another diagnostic that pertains to the depth of the convection zone is the photospheric lithium abundance. Lithium is underabundant in the Sun, but if the convection zone were as deep as about $2.6 \mathrm{x}$ $10^{5} \mathrm{~km}$, almost all the solar lithium would have been destroyed by nuclear reactions. The estimate from high-degree five-minute oscillations is easily within this limit.

\section{Five-minute Oscillations of Low Degree}

These are acoustic waves of high order, many of which penetrate to the centre of the Sun. The pioneering work by G.R. Isaak and his colleagues at the University of Birmingham demonstrated that the oscillations can be measured from Doppler shifts of spectral lines in light integrated from the whole or substantial fractions of the solar disk. The frequencies are sufficiently well separated that individual modes can be isolated in power spectra. This was first achieved by E. Fossat, G. Grec and M. Pomerantz, who obtained 120 hours of continuous data by observing the Sun from the South Pole.

The most basic property these modes centre to the surface of the Sun. The reciprocal of this quantity is roughly twice the mean frequency separation between modes of like degree. A direct comparison with standard theory implies a model relatively rich in helium and heavy elements, and consequently quite a deep convection zone, in accord with the dictates of the modes of high degree. Though some of these modes do penetrate to the centre of the Sun, their frequencies are determined mainly by the structure of the outer layers. In particular, frequencies are rather insenmeasure is the sound travel time from the 


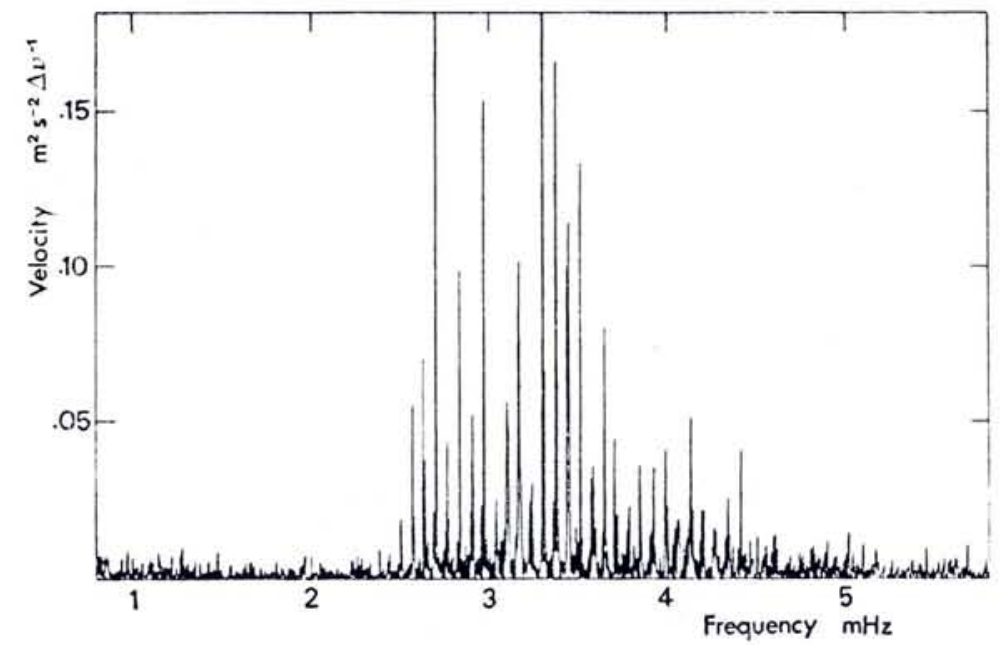

Fig. 2 - Power spectrum of integrated Doppler shifts of $120^{\mathrm{h}}$ continuous data obtained at the South Pole by G. Grec, E. Fossat and M. Pomerantz (Nature, 288 (1980) 541). The almost uniform/y spaced peaks are composed of groups of modes of alternately odd and even degrees. The spacing is roughly four times the sound travel time from the centre to the surface of the Sun.

sitive to conditions where the neutrinos are produced.

It must be pointed out that it has not yet been possible to obtain perfect agreement between theory and observation. Therefore one must be cautious in drawing conclusions from the analysis.

\section{Rotational Splitting of Modes of Low Degree}

The rotation of the Sun causes standing waves to precess, which is manifest as a frequency splitting of otherwise degenerate modes of like degree. The magnitude of the splitting is an average of the angular velocity in the solar interior, that average being weighted differently for different modes. Thus a knowledge of the splitting for a sufficiently wide selection of modes could teach us about the distribution of angular momentum within the Sun.

The first hint of rotational splitting was obtained by the Birmingham group. It was announced at the IAU Colloquium 66, which was held at the Crimean Astrophysical Observatory last September. The results are difficult to interpret, because modes that should not be detectable appear to be present in the data. Therefore we are not yet sure what the measurements imply.

More recently, rotational splitting appears to have been detected in oscillations of the limb-darkening function observed by R. Bos and H.A. Hill at the University of Arizona. Acoustic modes and internal gravity modes of low order and low degree have been measured, and from the splitting it has been possible to estimate the solar rotation. The angular velocity rises, probably monotonically, with depth, and in the core could be seven times the surface value. The results permit a rough estimate of $J_{2}$, the quadrupole moment of the external gravitational field. The value is about
$6 \times 10^{-6}$, and is just sufficient to be relevant to observational determinations of the parameters appearing in post-Newtonian representations of the gravitational field.
The Sun is not a dull sphere of luminous gas, it is active and variable over a considerable range of spatial and temporal scales. Solar activity shows up in the appearance and decay of sunspot groups and bright facular regions, the formation, fading or eruption of prominences, the explosion of solar flares, one of which releases as much energy as would be produced by a $1000 \mathrm{MW}$ power plant working for millions of years. Solar activity influences the earth through changes in the Sun's luminosity, by modulation of the solar wind and its interaction with the earth's magnetic field, and by energetic particles as well as UV radiation changing the conditions in the upper atmosphere.

Superimposed on all small scale spatial and temporal fluctuations is a quasiperiodic time modulation of activity with a period of roughly 11 years, the solar cycle. Fig. 1 shows the variation of the mean sunspot number (taken as a convenient measure of solar activity) from 1750 until today; the 11 year cycle and a modulation on a longer timescale is easily discernible. The variation of the ${ }^{14} \mathrm{C}$ production rate with solar activity allows the solar cycle to be traced back 8000 years into the past through the analysis of tree rings. Throughout this time the cycle continued to operate, apparently disturbed by a number of periods of very low activity.

\section{Future Prospects}

Aside from neutrino observations, the most likely means by which we might probe the solar interior is seismological. Already we have inferred that the Sun has a deeper convection zone than had previously been believed, and that the core of the Sun is possibly rotating up to seven times faster than the photosphere. Theoretical experiments have revealed that with not much more data than is presently available, it would be possible to measure the density stratification throughout the Sun to an accuracy of one or two per cent. Thus we are offered the possibility of measuring the inside of a star with a precision that has never before been envisaged.

\section{FURTHER READING}

1. Bahcall J.N., Solar neutrinos: theory versus experiment. Space Science Reviews, 24 (1979) 227-251.

2. Non-radial and Non-linear Stellar Pulsation, Eds. H.A. Hill and W.A. Dziembowski, Lecture Notes in Physics Vol 125, (Springer, Heidelberg) 1980.

3. "Physics of Solar Variations", Ed. V. Domingo, Proc. 14th ESLAB Symposium, Solar Physics, 74 (1981) 1 and 2.

\title{
Origin of the Solar Cycle
}

\author{
Manfred Schüssler, Göttingen \\ (Universitäts - Sternwarte)
}

Activity is concentrated into two belts in latitude, one on each hemisphere, which drift from mid latitudes towards the solar equator in the course of the cycle. It has become clear that solar activity is of magnetic origin and can only be understood by treating the electrodynamics of electrically conducting gases, i.e. plasmas. Let me stress a few of the most important results that any theoretical model must accommodate:

- The polar magnetic fields of the Sun change their magnetic polarity around activity maxima. Consequently, the magnetic period of the solar cycle is 22 years. This is supported by observation of the polarities of sunspot groups ("polarity rules").

- There is a small-scale component of the distribution of active regions which is not confined to the activity belts and seems to vary in antiphase with the big active regions while containing at least the same amount of magnetic flux.

- The non-spot magnetic fields are concentrated into narrow "flux tubes" with high flux density (100-200 mT in the solar photosphere).

- The brightness of sunspots varies during the cycle, darker spots appearing predominantly around activity maxima, brighter spots around activity minima. 